\title{
Soluble membrane attack complex is diagnostic for intraventricular shunt infection in children
}

\author{
Theresa N. Ramos', Anastasia A. Arynchyna², Tessa E. Blackburn', Scott R. Barnum ${ }^{1,3}$, \\ and James M. Johnston² \\ 'Department of Microbiology, '2Division of Pediatric Neurosurgery, Department of Neurosurgery, Children's of Alabama, \\ ${ }^{3}$ Department of Neurology, University of Alabama at Birmingham (UAB), Birmingham, Alabama, USA.
}

\begin{abstract}
BACKGROUND. Children treated with cerebrospinal fluid (CSF) shunts to manage hydrocephalus frequently develop shunt failure and/or infections, conditions that present with overlapping symptoms. The potential life-threatening nature of shunt infections requires rapid diagnosis; however, traditional microbiology is time consuming, expensive, and potentially unreliable. We set out to identify a biomarker that would identify shunt infection.
\end{abstract}

METHODS. CSF was assayed for the soluble membrane attack complex (sMAC) by ELISA in patients with suspected shunt failure or infection. CSF was obtained at the time of initial surgical intervention. Statistical analysis was performed to assess the diagnostic potential of sMAC in pyogenic-infected versus noninfected patients.

RESULTS. Children with pyogenic shunt infection had significantly increased sMAC levels compared with noninfected patients $(3,211 \pm 1,111 \mathrm{ng} / \mathrm{ml}$ vs. $26 \pm 3.8 \mathrm{ng} / \mathrm{ml}, P=0.0001)$. In infected patients undergoing serial CSF draws, sMAC levels were prognostic for both positive and negative clinical outcomes. Children with delayed, broth-only growth of commensal organisms ( $P$. acnes, $S$. epidermidis, etc.) had the lowest sMAC levels $(7.96 \pm 1.7 \mathrm{ng} / \mathrm{ml})$, suggesting contamination rather than shunt infection.

CONCLUSION. Elevated CSF sMAC levels are both sensitive and specific for diagnosing pyogenic shunt infection and may serve as a useful prognostic biomarker during recovery from infection.

FUNDING. This work was supported in part by the Impact Fund of Children's of Alabama.

Conflict of interest: T.N. Ramos, S.R. Barnum, and J.M. Johnston are coinventors on a pending international patent related to immunodiagnostics for complement biomarkers. S.R. Barnum serves on the scientific advisory board for Achillion Pharmaceuticals. J.M. Johnston is a coinventor on a pending international patent related to the prevention of head injury. This research was supported in part by the Impact Fund of Children's of Alabama.

Submitted: April 13, 2016 Accepted: May 31, 2016 Published: July 7, 2016

Reference information: JCI Insight. 2016;1(10):e87919. doi:10.1172/jci.insight.87919.

\section{Introduction}

Hydrocephalus, characterized by disordered secretion, circulation, and/or absorption of cerebrospinal fluid (CSF), is estimated to afflict 1 million people in the US. Infant hydrocephalus, occurring in 1 in every 1,000 births, arises from hemorrhage due to premature birth, intrauterine or postpartum infection, genetic syndromes, neoplasms, or ventricular/vascular malformations $(1,2)$. Placement of ventricular shunts is the most common procedure performed for the management of hydrocephalus but is unfortunately characterized by significant associated morbidity, including occlusion, breakage, and infection. Health expenditures associated with CSF shunting procedures are estimated to cost $\$ 2$ billion per year in the US $(2,3)$. Shunt failure rates remain high, as assessed in a recent international study that documented 30-day and 1-year shunt failure rates of $12.9 \%$ and $28.8 \%$, respectively (4), and institutional shunt infection rates range from $5 \%-12 \%$ in modern published cohorts $(5,6)$. Although mortality from shunt surgery is low $(0.5 \%)$, the long-term mortality rate remains high $(7,8)$. Current protocols for diagnosis of shunt malfunction or infection rely on costly radiographic imaging and traditional microbiological methods that often result in unnecessary hospitalization and treatment with antibiotics, leading to substantial healthcare costs. Given the high complication rate of shunt placement and limitations of the existing evaluation protocol, new methods for diagnosis of shunt infection based on advances in modern immunology would potentially improve the quality and efficiency of care for patients with hydrocephalus, leading to improved outcomes with significant cost savings by avoiding unnecessary hospitalization and treatment.

Implanting shunt hardware elicits an immune response due to surgically induced trauma and, in many cases, to external and intrathecal bacterial colonization of the tubing and other shunt componentry. 
Table 1. Patient Inclusion/exclusion criteria

\begin{tabular}{cc}
\hline Inclusion Criteria & Exclusion Criteria \\
\hline Age $\leq 18$ years & Started on antibiotics prior to draw \\
CSF draw via shunt tap, OR draw, EVD draw & Shunt infection within the last 180 days \\
Diagnosis of hydrocephalus & Newly diagnosed tumors \\
Shunt presence or ETV procedure & Ventricular irrigation \\
OR, operating room; EVD, extraventricular device; ETV, endoscopic third ventriculostomy.
\end{tabular}

Intraventricular infection will lead to potent immune responses, including the activation of resident glial cells, signaling through toll-like receptors, release of cytokines and chemokines, and recruitment and activation of myeloid cells (macrophages, neutrophils, and other granulocytes) $(9,10)$. In addition, subclinical and repeated infections lead to the generation of antibodies to the pathogenic organism(s), bacterial biofilms, and silicone elastomers in the shunt componentry $(11,12)$. Complement likely plays an important role in this clinical setting, but its role in the development and progression of the inflammatory response in shunt infections remains ill defined. Complement proteins are produced within the CNS by astrocytes, microglia, and neurons, and inflammatory cytokines readily augment intrathecal production (13-17). In bacterial meningitis, traumatic brain injury, and ischemic stroke, the level of complement proteins and activation fragments in CSF or the level deposited on tissue is markedly elevated, suggesting that local complement production and activation could contribute significantly to shunt infection immunopathology (18-22). In a study examining immune complex disease in shunt infections, serum complement levels were depressed, indicating complement activation, but this did not address the role of complement at the site of infection (23). More recently, the complement inhibitor vitronectin and activated C9 were shown to be present on ventriculoperitoneal shunts and ventricular drainage catheters, suggesting a role for complement in the pathogenesis of shunt infections (24).

In the present report, we undertook studies to determine if the soluble membrane attack complex (sMAC) could serve as an immune biomarker to diagnose bacterial infection in children with ventriculoperitoneal shunts. The MAC is a large macromolecular protein complex composed of 5 complement proteins ( $\mathrm{C} 5 \mathrm{~b}, \mathrm{C} 6, \mathrm{C} 7, \mathrm{C} 8$, and $\mathrm{C} 9$ ) that together generate a pore-forming structure capable of lysing bacteria and other microorganisms $(25,26)$. The MAC is formed when the complement terminal pathway is activated through any of the early activation pathways (alternative, classical, or lectin) or the extrinsic protease pathway (27-29). Membrane attack complexes that form in the fluid phase interact with the complement regulatory proteins clusterin or vitronectin, forming a soluble complex no longer able to bind to pathogen or host tissue membranes (29). The MAC can also contribute to inflammation through production of ion fluxes and activation of proinflammatory signaling pathways in host cells and, through these mechanisms, serve to exacerbate the severity of shunt infections (30). Soluble MAC in normal CSF or noninflammatory CNS disease is low or undetectable but increases in concentration in subarachnoid hemorrhage, traumatic brain injury, and autoimmune diseases (31-35). We show here that sMAC levels in the CSF of patients with pyogenic shunt infections were elevated over 100-fold compared with noninfected controls or patients with delayed, broth-only growth commensal infections. On subsequent shunt taps, sMAC demonstrated prognostic value for patient outcome. Our data suggest that sMAC levels above a $43 \mathrm{ng} / \mathrm{ml}$ cutoff are a sensitive (93\%) and specific (86\%) biomarker with excellent diagnostic capability (receiver operator characteristic [ROC] AUC 0.966, $P<0.0001$ ) for identifying pyogenic infections in shunt patients.

\section{Results}

Study Description. For this study, we quantitated ventricular CSF sMAC levels in the pediatric population at Children's of Alabama, which included patients under evaluation for newly diagnosed hydrocephalus, shunt failure, and/or infection (246 patients) (Figure 1). Based on clinical presentation, laboratory findings, and imaging data, patients were divided into the following groups: (i) infection caused by pyogenic organisms, (ii) infection associated with commensal organisms, and (iii) noninfection based on clinical symptoms, microbiology, and laboratory markers of infection. Children treated with antibiotics prior to shunt tap (because of the possible effect antibiotics could have on complement activation), shunt infection within the previous 180 days (to insure that we had a cohort clear of residual infection and that 


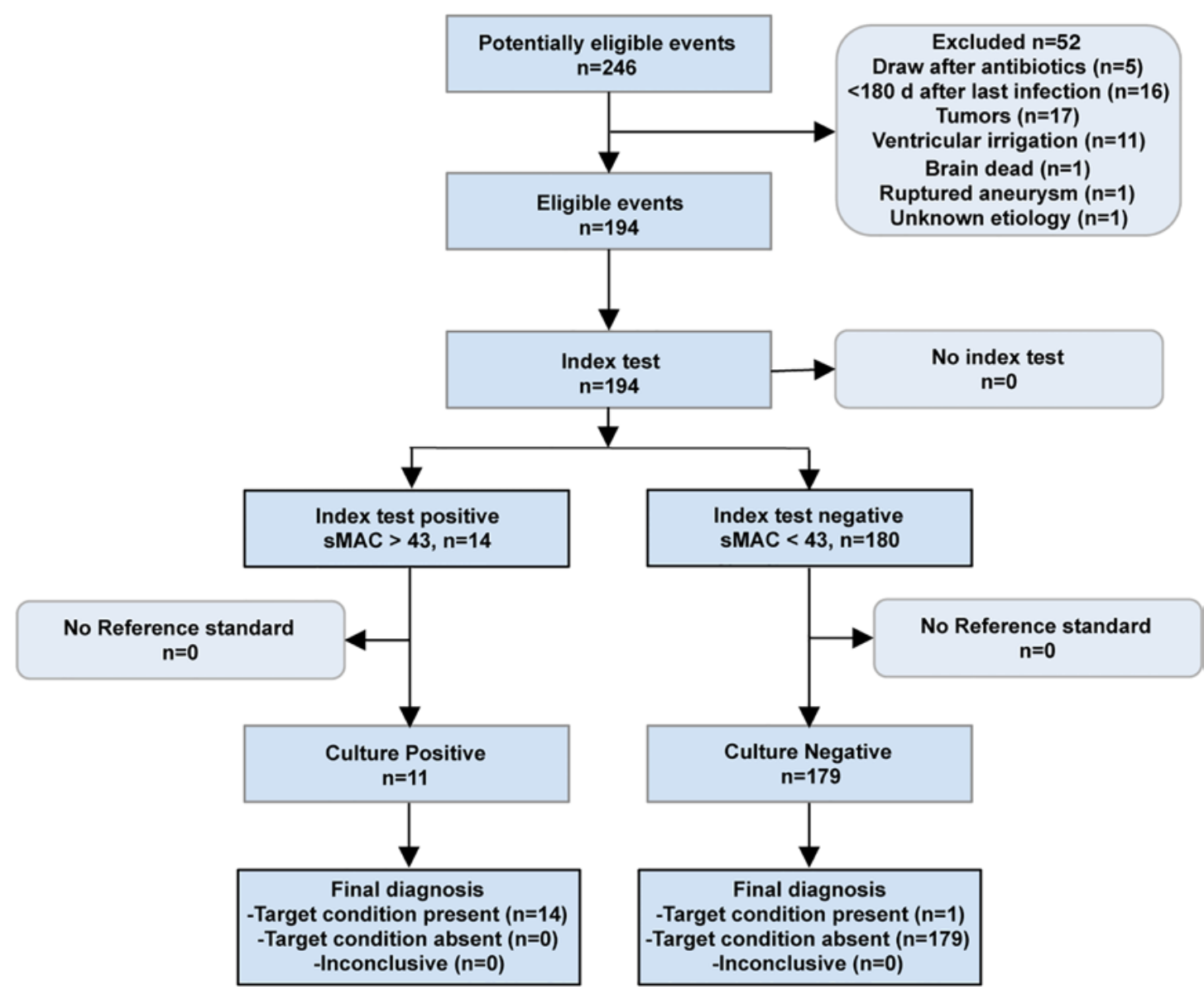

Figure 1. CONSORT flow diagram.

residual sMAC levels would have returned to baseline), and children with newly diagnosed tumors were excluded from this analysis (Table 1). Patients with newly diagnosed tumors were excluded from analysis due to potential confounding effects of malignant tumors on complement activation $(36,37)$. Patient demographics are shown in Table 2.

Soluble MAC levels are elevated in patients with pyogenic shunt infections compared with noninfected patients. Baseline soluble MAC levels in the CSF of uninfected individuals (pediatric and adult) (CSF free of culturable bacteria and enterovirus, herpes simplex virus types 1 and 2, or Epstein Barr virus as detected by PCR, no fever, normal levels of WBC, protein, and glucose) ranged from undetectable to approximately 5-10 ng/ml (data not shown). In contrast, we found that sMAC levels were markedly elevated in patients with culture-confirmed pyogenic shunt infection $(3,211 \pm 1,111 \mathrm{ng} / \mathrm{ml}, n=15)$ (Figure 2). Soluble MAC levels correlated well with changes in CSF protein levels and WBC number $(\mathrm{r}=0.77$ and 0.68 and $P=$ 0.0006 and 0.004 , respectively, Spearman's correlation) (Table 3). These parameters in premature infants with intraventicular hemorrhage were not significantly different when compared with parameters of older children (data not shown). The majority of the shunt infections were caused by $S$. aureus, some of which were methicillin resistant strains. Additional pathogens identified in the CSF of this patient group included Enterococcus faecalis, E. coli, S. caprae, Proteus mirabilis, Pseudomonas aeruginosa, and S. epidermidis. In noninfected patients, CSF sMAC levels were over 120-fold lower than those with pyogenic infection $(n=224$, $26 \pm 3.8 \mathrm{ng} / \mathrm{ml}$ vs. $3,211 \pm 1,111 \mathrm{ng} / \mathrm{ml}, P=0.0001,1$-way ANOVA) (Figure 2). This patient cohort had slightly elevated WBC and protein levels (Table 3), possibly due to previous hemorrhage, shunt irritation, or other shunt-related issues. Interestingly, a small cohort of patients with Staphylococcus (epidermidis, caprae, capitis, or hominis) or $P$. acnes infection had low or undetectable levels of sMAC on the first shunt tap $(7.96 \pm 1.67 \mathrm{ng} / \mathrm{ml}, n=9, P=0.0001,1$-way ANOVA) (Figure 2) and had no significant increase in sMAC levels on subsequent taps, if they were obtained (data not shown). Growth of these organisms occurred in 
Table 2. Patient Demographics

\begin{tabular}{|c|c|c|c|c|}
\hline & ALL $(n=248)$ & Pyogenic $(n=15)$ & Commensals $(n=9)$ & NonInfected $(n=224)$ \\
\hline Years & $4.36 \pm 5.69$ & $2.67 \pm 4.1$ & $7.78 \pm 7.5$ & $4.34 \pm 5.6$ \\
\hline Range - yr & $0-18$ & $0-13$ & $0-17$ & $0-18$ \\
\hline Range - mo & $0-221$ & $1-156$ & $1-204$ & $0-221$ \\
\hline \multicolumn{5}{|l|}{ Sex } \\
\hline \multicolumn{5}{|l|}{ Race or ethnicity } \\
\hline Mixed European descent & 140 (56.5\%) & $8(53.3 \%)$ & $4(44.4 \%)$ & $128(57.1 \%)$ \\
\hline Black & $106(42.1 \%)$ & $7(46.7 \%)$ & $5(55.6 \%)$ & $94(42 \%)$ \\
\hline Other & $2(0.8 \%)$ & - & - & $2(0.9 \%)$ \\
\hline \multicolumn{5}{|l|}{ Etiology } \\
\hline Aqueductal Stenosis & $15(6 \%)$ & - & - & $15(6.7 \%)$ \\
\hline Encephalocele & $12(4.8 \%)$ & $1(6.7 \%)$ & - & $11(4.9 \%)$ \\
\hline Spontaneous IVH & $11(4.4 \%)$ & - & - & $11(4.9 \%)$ \\
\hline Cephalic disorder & $9(3.6 \%)$ & $1(6.7 \%)$ & $2(22.2 \%)$ & $6(2.7 \%)$ \\
\hline Trauma & $9(3.6 \%)$ & $1(6.7 \%)$ & $1(11.1 \%)$ & $7(31 \%)$ \\
\hline Prematurity without IVH & $8(3.2 \%)$ & - & $3(33.3 \%)$ & $5(2.2 \%)$ \\
\hline Cyst & $6(2.4 \%)$ & - & - & $6(2.7 \%)$ \\
\hline Dandy-Walker & $6(2.4 \%)$ & - & - & $6(2.7 \%)$ \\
\hline Postinfectious & $6(2.4 \%)$ & - & - & $6(2.7 \%)$ \\
\hline Tumor & $6(2.4 \%)$ & $2(13.3 \%)$ & - & $4(1.8 \%)$ \\
\hline Obstructive hydrocephalus & $2(0.8 \%)$ & - & - & $2(0.9 \%)$ \\
\hline
\end{tabular}

broth only, and these patients did not have elevated WBC counts or protein levels associated with bacterial infection (Table 3). Only 1 of the 9 patients in this cohort had a fever $\left(\geq 38.3^{\circ} \mathrm{C}\right)$ at the time of the shunt tap. These results raise the possibility that contamination with these commensal organisms occurred during the shunt tap or subsequent sample handling rather than being true shunt-related infections.

To assess the biomarker potential for sMAC to discriminate between pyogenic infection and noninfected controls, we performed a ROC analysis and calculated the sensitivity and specificity, as well as positive and negative predictive values (Figure 3). ROC analysis demonstrated that sMAC has excellent diagnostic capability for discriminating pyogenic infection from noninfection in shunt patients (area $=0.966,95 \% \mathrm{CI}$, 0.934-0.99, $P<0.0001)$. Using the maximum paired sensitivity and specificity values from the ROC analysis, we determined a cutoff value of $\sim 43 \mathrm{ng} / \mathrm{ml}$ for sMAC levels in detecting pyogenic shunt infection. Applying this cutoff to our pyogenic infection cohort, we detected 14 of 15 infections based on the final clinical diagnosis of these patients. Soluble MAC was both sensitive and specific for diagnosing pyogenic shunt infections $(93.3 \%, 95 \%$ CI, 68.1\%-99.8\% and 86.6\%, 95\% CI, 81.5\%-90.7\%, respectively) (Table 4). Although the negative predictive value was high (99.5\%, 95\% CI, 97.3\%-99.9\%), the positive predictive was low $(31 \%, 95 \%$ CI, $18.2 \%-46.7 \%)$ because of the number of false positives in the symptomatic hydrocephalus patient cohort. The current diagnostic gold standard of bacterial culture was less accurate, detecting only 11 of 15 pyogenic infections in this series. Culture results were less sensitive (73.3\% vs. 93.3\%) but comparably specific (95.5\% vs. $86.6 \%$ ) compared with sMAC. For bacterial culture, the negative predictive value was also high (98.2\%, 95\% CI, 95.4\%-99.5\%), while the positive predictive was low $(52.4 \%, 95 \%$ CI, $29.8 \%$ $74.3 \%$ ). In contrast, the positive predictive value for WBC count and protein levels were very low (14.3\% and $9.4 \%$, respectively), but both had good negative predictive value ( $99 \%$ and $98 \%$, respectively). Gram staining 


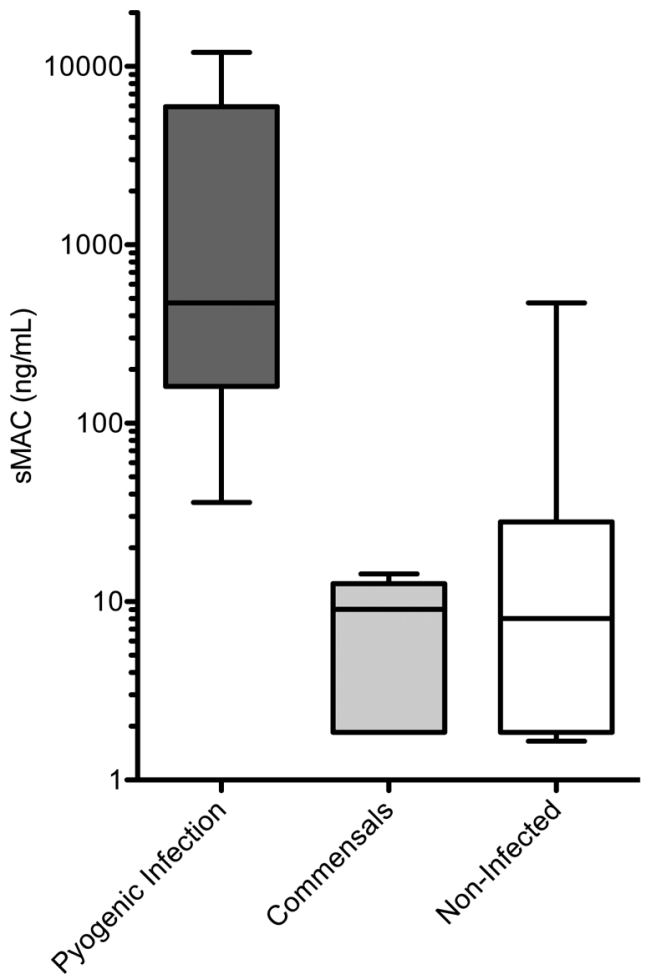

Figure 2. Cerebrospinal fluid sMAC levels in uninfected children or children infected with pyogenic or commensal organisms. Cerebrospinal fluid samples were obtained via the external ventricular drain and assayed in duplicate for sMAC levels using the Quidel sC5-9 ELISA. sMAC levels were compared between uninfected $(n=230)$, commensal-infected $(n=9)$, and pyogenic-infected $(n=15)$ patients. Shown are box and whisker plots for each group; the upper and lower bars represent the maximum and minimum values. The box represents the first and third quintiles, and the line in the box represents the median value. Mean values between patient groups were compared using 1-way ANOVA with Kruskal-Wallis test and Dunn's multiple comparison test.

was the least accurate method for detecting bacterial infection in shunt infection, with 5 false negatives and only $72 \%$ sensitivity.

Soluble MAC levels have prognostic value in monitoring patients with pyogenic infections. To discriminate between shunt infection and failure, many patients have repeated shunt taps performed in an effort to determine the presence and identity of potential pathogens and to assess clinical parameters associated with infection. Analysis of CSF MAC levels in patients from both groups revealed that SMAC levels decline in some shunt infec-

tion patients in a manner that correlates with their clinical recovery. In 3 representative patients, we observed that sMAC levels decline 50\% within 4-5 days after the start of antibiotic treatment and then return to normal or near normal levels in a patient-dependent fashion (Figure 4, A-C). Conversely, in patients responding poorly to antibiotics and other supportive therapy, sMAC levels fail to decline and tend to increase over time, correlating with the severity of the clinical picture (Figure 4, D and E). These data suggest that repeated measuring of sMAC in shunt-infection patients offers a new and sensitive method to monitor patient progress during the management of shunt infection. In contrast, sMAC levels in patients with shunt failure often increased over the first 5 days of monitoring (10\%-25\% over the value of first tap) and then varied widely from patient to patient over time (Figure 4, F-H). The ability of sMAC levels to discriminate between shunt infection and failure is readily evident when comparing the changes in sMAC levels over time for patient 126 . This patient initially presented with classic symptoms of shunt failure, including bulging fontanels and split sutures. The sMAC levels were modestly elevated ( $<100 \mathrm{ng} / \mathrm{ml}$ at all time points) and varied widely over nearly 100 days of monitoring (Figure $4 \mathrm{H})$. This patient subsequently developed a shunt infection and presented with elevated sMAC levels $(>1,400$ $\mathrm{ng} / \mathrm{ml}$ on first tap) (Figure 4C). Soluble MAC levels then declined rapidly after the onset of antibiotic treatment.

\section{Discussion}

Hydrocephalus is a disorder of CSF physiology that develops due to an imbalance in the rate of production and reabsorption of CSF that arises as a result of multiple causes, including congenital malformation, intraventricular hemorrhage (commonly seen in premature infants), infection, trauma, tumors, and cysts (1, 2, 38). Standard treatment of hydrocephalus, by surgical insertion of an in-dwelling ventriculoperitoneal

Table 3. Comparison of clinical lab parameters

\begin{tabular}{lccc}
\hline Patient Cohort & WBC $^{\text {A }}$ & Protein $^{\mathbf{B}}$ & Glu $^{\mathbf{c}}$ \\
Pyogenic Infection & $4,527 \pm 3,313$ & $1,392 \pm 512$ & $32 \pm 4.9$ \\
Commensals & $3.67 \pm 1.43$ & $46.8 \pm 12.9$ & $52.3 \pm 6.8$ \\
Noninfected & $12.3 \pm 2.3$ & $123.5 \pm 13.3$ & $49.1 \pm 1.1$
\end{tabular}

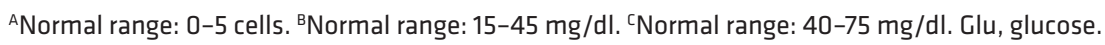


shunt, is effective in diverting excess CSF from the ventricles to the peritoneal cavity. Nonetheless, shunt failure arising from breakage, migration, or occlusion of shunt componentry - or from infection - is a frequent event $(6,10,39,40)$. Diagnosing shunt failure and infection in the pediatric population is often challenging because of overlapping clinical findings and poor predictive values for common signs between the two conditions $(2,41)$. Aside from the medical history and clinical exam, diagnosis of shunt failure versus infection is facilitated through imaging (CT, radionuclide, MRI, functional MRI), shunt taps to examine for abnormal CSF pressure, and culture for infectious organisms $(39,40,42,43)$. Inflammatory biomarkers including C-reactive protein, IL- 6 , IL- 1 , and TNF- $\alpha$ have been assessed for their utility in detecting shunt infection (23, 44-48). These studies, for the most part, quantitated serum levels - not CSF levels - of these immune mediators, limiting their value as a diagnostic for CNS infection. Of the cytokines, IL-6 had the high-

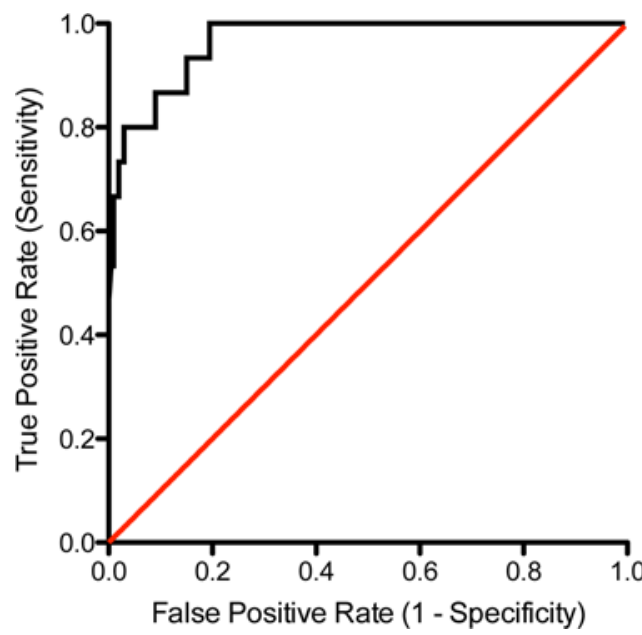

Figure 3. Diagnostic performance of sMAC for pyogenic shunt infection. ROC curves were plotted using data from the pyogenic and noninfected patient cohorts to assess the performance characteristic of sMAC for shunt infection. The AUC was 0.996 with a $95 \% \mathrm{Cl}$ of $0.934-0.99$. est diagnostic accuracy in one study (45) but was of limited value in infants with posthemorrhagic hydrocephalus (46). More recently, CSF protein biomarkers - including amyloid precursor protein, neural cell adhesion molecule-L1, and neural cell adhesion molecule-1 - have shown diagnostic promise in posthemorrhagic hydrocephalus, common in preterm infants (49).

The results we report here demonstrate that SMAC is a sensitive and specific biomarker for diagnosing shunt infections. Soluble MAC levels in pyogenic infections were approximately 100 -fold higher on average than those in noninfected patients, a cohort that includes patients with symptomatic hydrocephalus or functioning shunts as assessed by clinical exam and MRI imaging. Soluble MAC levels correlated well with changes in CSF protein levels and WBC count, two laboratory parameters routinely used in the diagnosis of CNS infection. In several patients, the sMAC level was more revealing than either protein or WBC levels, suggesting that sMAC is a better diagnostic tool. Although there was wide variation in sMAC levels within the infected patient cohort, we speculate that this is most likely a function of the duration and severity of the infection. This possibility is supported by data in a subset of patients whose sMAC levels were higher on subsequent shunt taps (data not shown). Remarkably, the sMAC level in the one false-negative patient in our pyogenic infected cohort increased nearly 5 -fold on the second tap obtained 3 day later $(36 \mathrm{ng} / \mathrm{ml}$ vs. $168 \mathrm{ng} / \mathrm{ml}$ ). Altogether, these data suggest that repeated monitoring of sMAC levels may have significant clinical value.

One possible outcome of our study was that sMAC levels in shunt infections could have pathogen-specific diagnostic value. However, there was no correlation with sMAC levels and any one particular organism in our infected patient cohort, nor was there a correlation between sMAC levels and Gram-positive versus Gram-negative organisms. Although our data indicate that sMAC levels are not yet useful as a tool to aid in pathogen subtyping, we observed that sMAC levels in patients whose CSF cultures grew commensal organisms including $P$. acnes and Staphylococcus spp. (epidermidis, caprae, capitis, or hominis) had,

Table 4. Comparison of sensitivity, specificity, and predictive values between sMAC and bacterial culture in diagnosing pyogenic shunt infection

\begin{tabular}{lcccc}
\hline Diagnostic Test & Sensitivity & Specificity & Positive Predicative Value & Negative Predicative Value \\
SMAC & $93.3 \%, 95 \% \mathrm{Cl}, 68.1 \%-99.8 \%$ & $86.6 \%, 95 \% \mathrm{Cl}, 81.5 \%-90.7 \%$ & $31 \%, 95 \% \mathrm{Cl}, 18.2 \%-46.7 \%$ & $99.5 \%, 95 \% \mathrm{Cl}, 97.3 \%-99.9 \%$ \\
Bacterial Culture & $73.3 \%, 95 \% \mathrm{Cl}, 44.9 \%-92.2 \%$ & $95.5 \%, 95 \% \mathrm{Cl}, 91.9 \%-97.8 \%$ & $52.4 \%, 95 \% \mathrm{Cl}, 29.8 \%-74.3 \%$ & $98.2 \%, 95 \% \mathrm{Cl}, 95.4 \%-99.5 \%$
\end{tabular}



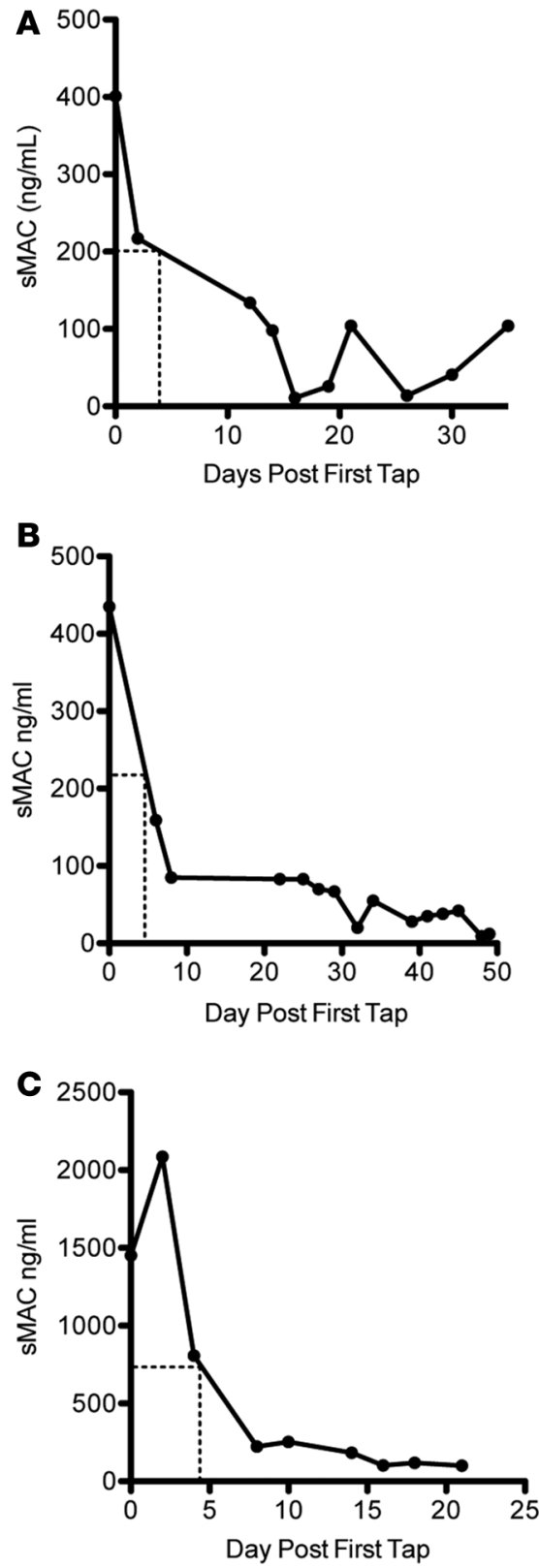
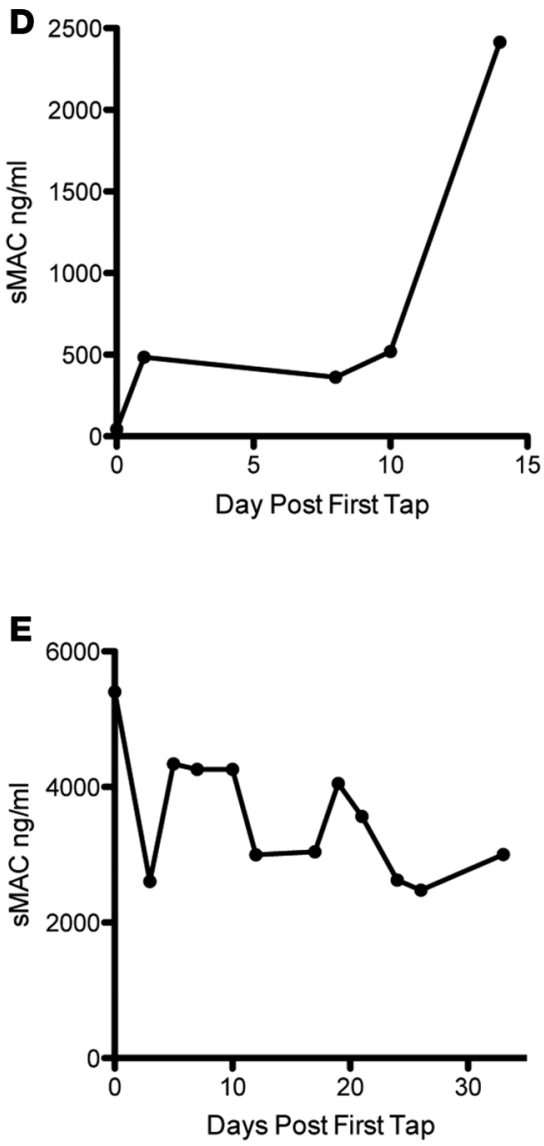
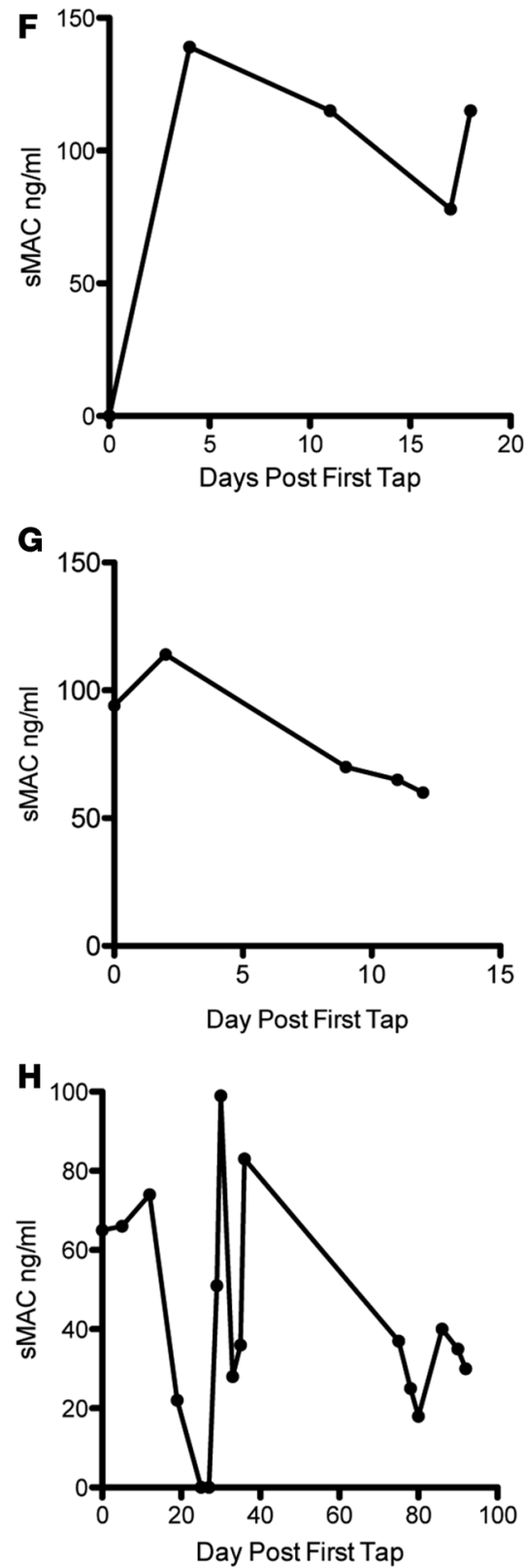

Figure 4. Cerebrospinal fluid sMAC levels decline rapidly in children with pyogenic infections after antibiotic administration. Serial CSF samples were obtained via the external ventricular drain and assayed for SMAC levels using the sC5-9 ELISA. The day 0 samples were obtained prior to administering antibiotics, while subsequent samples were collected after initiation of antibiotic treatment. In patients responsive to antibiotic and supportive therapy, the sMAC levels dropped to $50 \%$ of their initial level within 4-5 days and returned close to baseline levels within 2 weeks (dotted lines) (A-C). In patients unresponsive to antibiotic therapy, CSF sMAC levels frequently increased after day 0 and rarely returned to baseline ( $\mathbf{D}$ and $\mathbf{E})$. In patients with symptomatic shunt failure as diagnosed by MRI imaging, the SMAC levels were markedly lower than in pyogenic infection and varied widely over the course of monitoring (F-H).

on average, remarkably low sMAC levels $(\sim 8 \mathrm{ng} / \mathrm{ml})$. These organisms are frequent opportunistic pathogens, particularly on medical devices such as prosthetic heart valves, joint prostheses, and shunts (50-54). However, given that the organisms grew out in broth only or from later shunt taps, it is possible that these patients did not have true meningitic shunt infections. The clinical data strongly support this interpretation, since all these patients had normal or near normal WBC counts and CSF protein and glucose levels. Furthermore, only 1 of the 9 patients in this cohort experienced fever. We cannot rule out that the low sMAC levels in commensal-infected shunt patients are due to differential expression of virulence genes by these organisms that modulate complement activation and function, especially as it relates to biofilm production 
or proteases that may inactivate or inhibit complement (54-57). It is also possible that sMAC levels in this cohort are depressed, in part, due to complement depletion, as reported in $S$. epidermidis infections in ventriculoatrial shunt patients (23); however, this seems less likely, as sMAC is stable relative to other complement activation products (58).

Not surprisingly, the presence of a shunt alone alters the baseline levels of sMAC. The level of sMAC in CSF of our noninfected shunt patient cohort was elevated several-fold compared with patients with no shunt or infection ( $26 \mathrm{ng} / \mathrm{ml}$ vs. $\leq 5 \mathrm{ng} / \mathrm{ml}$ ). Activated C9 (MAC) and the complement regulatory protein vitronectin have been found on the surface of shunt devices and temporary ventricular drainage catheters (24). Complement deposition in this setting arises, in part, from biomaterial-induced activation of complement, which occurs on all tubing and plastics used in implanted medical devices (59). Complement activation may also increase when shunt componentry is damaged or degraded in situ over time, exposing new activating surfaces. Shunt migration may irritate ventricular tissue, leading to local inflammation and activation of complement. In addition, shunt migration may result in localized hemorrhage and activation of complement through the extrinsic protease pathway $(27,28)$. Interestingly, while $33 \%$ of uninfected patients with symptomatic hydrocephalus had undetectable sMAC levels, the remaining patients had, on average, low sMAC levels $(44.6 \pm 7.4 \mathrm{ng} / \mathrm{ml})$, suggesting a previously undescribed relationship between ventricular stretch and complement activation. Under the inflammatory conditions of a bacterial infection, local complement production by glial cells (ependymal cells, astrocytes, and microglia) and neurons may contribute substantially to intrathecal complement levels $(14,60-62)$. Furthermore, regional blood brain barrier disruption due to inflammation allows blood-derived complement to reach the ventricles. This combination of mechanisms undoubtedly accounts for the elevated levels of CSF sMAC we report here for the functioning shunt population and those with shunt infections.

In summary, our data indicate that monitoring the CSF levels of sMAC could be a powerful tool in the diagnosis and management of shunt infections. Patients with shunt failure or shunt infection often present with clinically overlapping symptoms. Given the risks and expenses associated with CNS infection and the invasive surgical procedures to revise or replace shunt componentry, a diagnostic that could — by itself or coupled with imaging - distinguish between these patient cohorts would significantly reduce healthcare costs and allow for more rapid and appropriate clinical care. Currently, the standard laboratory tests to identify bacterial infection (CSF cell counts, protein and glucose levels, Gram stain, and bacterial culture) cost over $\$ 1,000$ per draw at most hospitals and clinics in the US. Many shunt patients are repeatedly tapped $(37 \%$ in the patient cohort presented here); some are only tapped only twice, but some are tapped as many as 10-15 times in an effort to detect the presence of infection and identify the pathogen so as to use the most appropriate antibiotic regimen. This clinical approach is confounded by the fact that, $25 \%-30 \%$ of the time, no organism is detected in culture $(10,40)$. Although this study was performed using ELISA, conversion to a lateral flow assay system would have obvious benefits in terms of both overall cost and time. A rapid sensitive and specific point-of-care diagnostic test to identify shunt infection could reduce the number of shunt taps performed and, depending on the clinical scenario, could potentially be used as a stand-alone prognostic test for some patients.

\section{Methods}

Patient Selection and CSF collection. One hundred ninety-eight consecutive patients at Children's of Alabama undergoing evaluation and treatment for newly diagnosed hydrocephalus, shunt infection, and versus malfunction were enrolled in the study. Patient inclusion/exclusion criteria are listed in Table 1. Samples of CSF (1 ml) were collected via shunt taps, intraoperative collections, and extraventricular device draws from emergency rooms and clinics, operating rooms, and patient floors, respectively. Intraoperative collections occurred during (i) shunt placements/revisions/removals through shunt catheter and (ii) endoscopic procedures through a working channel. Irrigation of ventricles and use of preoperative antibiotics were recorded.

Study samples were collected at the same time as routine lab specimens to provide corresponding lab results for each specimen (glucose, protein, WBC, RBC, gram stain, and culture). Samples were labeled and stored at $-20^{\circ} \mathrm{C}$ and $/$ or $-80^{\circ} \mathrm{C}$ for a period of 1 week on average prior to analysis. Demographics, labs, patient diagnosis, and clinical signs of disease were recorded for each new event. Consecutive events were recorded. An event was defined as a new admission, clinic, or emergency room visit. By definition, one patient could have multiple events.

Soluble MAC quantitation. Soluble MAC was quantitated using the MicroVue complement sC5b-9 Plus enzyme immunoassay (Quidel Corporation) according to the manufacturers instructions. Samples were 
diluted as required in specimen diluent, and sMAC levels were calculated from a standard curve including internal high and low controls. The lower limit of detection for the assay was $3.7 \mathrm{ng} / \mathrm{ml}$. Samples below the lower limit of detection were assigned a value one-half lower than the limit of detection. Samples were assayed blinded to patient diagnosis.

Statistics. CSF samples were run in duplicate, and sMAC levels were plotted in a box and whisker plot format using Graphpad Prism 6. Mean values between patient groups were compared using 1-way ANOVA with Kruskal-Wallis test and Dunn's multiple comparison test. $P<0.05$ was considered significant. Associations between continuous factors were assessed using the Spearman's correlation coefficient. The relative ability of SMAC to indicate a pyogenic infection was estimated by calculating the area under ROC curves using Graphpad Prism 6 and compared noninfected and pyogenic infected patients. Data points were excluded if they were $>10 \pm$ SD from the mean value of the group for nonformally distributed data.

Study approval. The patient cohort consisted of hydrocephalus patients treated the Children's of Alabama. All participants provided written informed consent before inclusion in the study. The IRB at UAB approved this study.

\section{Author contributions}

TNR, SRB, and JMJ created the concept and design of the study. AAA, TNR, and TEB acquired samples and managed the data. TNR, AAA, TEB, SRB, and JMJ analyzed and interpreted the data. TNR, AAA, $\mathrm{TEB}, \mathrm{SRB}$, and JMJ wrote, reviewed, and revised the manuscript.

\section{Acknowledgments}

The authors would like to thank Jeffery Blount, Jerry Oakes, Brandon Rocque, and Curtis Rozzelle for their support and help with sample acquisition throughout the study. The authors also acknowledge the support of Fran Lund (Department of Microbiology, UAB), Mitch Cohen (Department of Pediatrics, UAB), and Michael Warren (Children's of Alabama).

Address correspondence to: James M. Johnston, Division of Pediatric Neurosurgery, Department of Neurosurgery, Children's of Alabama, University of Alabama at Birmingham, 1600 7th Avenue S., Lowder 400, Birmingham, Alabama 35233, USA. Phone: 205.638.9653; E-mail: James.Johnston@childrensal.org.

1. Tully HM, Dobyns WB. Infantile hydrocephalus: a review of epidemiology, classification and causes. Eur J Med Genet. 2014;57(8):359-368.

2. Kahle KT, Kulkarni AV, Limbrick DD Jr, Warf BC. Hydrocephalus in children. Lancet. 2016;387(10020):788-799.

3. Simon TD, et al. Hospital care for children with hydrocephalus in the United States: utilization, charges, comorbidities, and deaths. J Neurosurg Pediatr. 2008;1(2):131-137.

4. Al-Tamimi YZ, et al. Ventriculoperitoneal shunt 30-day failure rate: a retrospective international cohort study. Neurosurgery. 2014;74(1):29-34.

5. Kestle JR, et al. A new Hydrocephalus Clinical Research Network protocol to reduce cerebrospinal fluid shunt infection. J Neurosurg Pediatr. 2016(4):361-366.

6. Wong JM, et al. Patterns in neurosurgical adverse events: cerebrospinal fluid shunt surgery. Neurosurg Focus. 2012;33(5):E13.

7. Smith ER, Butler WE, Barker FG 2nd. In-hospital mortality rates after ventriculoperitoneal shunt procedures in the United States, 1998 to 2000: relation to hospital and surgeon volume of care. J Neurosurg. 2004;100(2 Suppl Pediatrics):90-97.

8. Vinchon M, Baroncini M, Delestret I. Adult outcome of pediatric hydrocephalus. Childs Nerv Syst. 2012;28(6):847-854.

9. Heidemann SM, Fiore M, Sood S, Ham S. Eosinophil activation in the cerebrospinal fluid of children with shunt obstruction. Pediatr Neurosurg. 2010;46(4):255-258.

10. Gutierrez-Murgas Y, Snowden JN. Ventricular shunt infections: immunopathogenesis and clinical management. J Neuroimmunol. 2014;276(1-2):1-8.

11. Goldblum RM, Pelley RP, O’Donell AA, Pyron D, Heggers JP. Antibodies to silicone elastomers and reactions to ventriculoperitoneal shunts. Lancet. 1992;340(8818):510-513.

12. VandeVord PJ, et al. Immune reactions associated with silicone-based ventriculo-peritoneal shunt malfunctions in children. Biomaterials. 2004;25(17):3853-3860.

13. Morgan BP, Gasque P. Extrahepatic complement biosynthesis: where, when and why? Clin Exp Immunol. 1997;107(1):1-7.

14. Stahel PF, Barnum SR. Bacterial meningitis: complement gene expression in the central nervous system. Immunopharmacology. 1997;38(1-2):65-72.

15. Nataf S, Stahel PF, Davoust N, Barnum SR. Complement anaphylatoxin receptors on neurons: new tricks for old receptors? Trends Neurosci. 1999;22(9):397-402.

16. Barnum SR. Complement in central nervous system inflammation. Immunol Res. 2002;26(1-3):7-13.

17. van Beek J, Elward K, Gasque P. Activation of complement in the central nervous system: roles in neurodegeneration and neuroprotection. Ann N Y Acad Sci. 2003;992: 56-71. 
18. Kossmann T, Stahel PF, Morganti-Kossmann MC, Jones JL, Barnum SR. Elevated levels of the complement components C3 and factor B in ventricular cerebrospinal fluid of patients with traumatic brain injury. J Neuroimmunol. 1997;73(1-2):63-69.

19. Stahel PF, et al. TNF-alpha-mediated expression of the receptor for anaphylatoxin C5a on neurons in experimental Listeria meningoencephalitis. J Immunol. 1997;159(2):861-869.

20. Stahel PF, Nadal D, Pfister HW, Paradisis PM, Barnum SR. Complement C3 and factor B cerebrospinal fluid concentrations in bacterial and aseptic meningitis. Lancet. 1997;349(9069):1886-1887.

21. Gasque P, et al. The receptor for complement anaphylatoxin C3a is expressed by myeloid cells and nonmyeloid cells in inflamed human central nervous system: analysis in multiple sclerosis and bacterial meningitis. J Immunol. 1998;160(7):3543-3554.

22. Arumugam TV, Woodruff TM, Lathia JD, Selvaraj PK, Mattson MP, Taylor SM. Neuroprotection in stroke by complement inhibition and immunoglobulin therapy. Neuroscience. 2009;158(3):1074-1089.

23. Bayston R, Rodgers J. Role of serological tests in the diagnosis of immune complex disease in infection of ventriculoatrial shunts for hydrocephalus. Eur J Clin Microbiol Infect Dis. 1994;13(5):417-420.

24. Lundberg F, et al. Presence of vitronectin and activated complement factor C 9 on ventriculoperitoneal shunts and temporary ventricular drainage catheters. J Neurosurg. 1999;90(1):101-108.

25. Sonnen AF, Henneke P. Structural biology of the membrane attack complex. Subcell Biochem. 2014;80:83-116

26. Serna M, Giles JL, Morgan BP, Bubeck D. Structural basis of complement membrane attack complex formation. Nat Commun. 2016;7:10587.

27. Amara U, et al. Molecular intercommunication between the complement and coagulation systems. J Immunol. 2010;185(9):5628-5636.

28. Huber-Lang M, et al. Generation of C5a in the absence of C3: a new complement activation pathway. Nat Med. 2006;12(6):682-687.

29. Merle NS, Church SE, Fremeaux-Bacchi V, Roumenina LT. Complement System Part I - Molecular Mechanisms of Activation and Regulation. Front Immunol. 2015;6:262

30. Morgan BP. The membrane attack complex as an inflammatory trigger. Immunobiology. 2015;221(6):747-751

31. Mollnes TE, Vandvik B, Lea T, Vartdal F. Intrathecal complement activation in neurological diseases evaluated by analysis of the terminal complement complex. J Neurol Sci. 1987;78(1):17-28.

32. Sanders ME, et al. Terminal complement complexes (SC5b-9) in cerebrospinal fluid in autoimmune nervous system diseases. Ann N Y Acad Sci. 1988;540:387-388.

33. Lindsberg PJ, et al. Complement activation in the central nervous system following blood-brain barrier damage in man. Ann Neurol. 1996;40(4):587-596.

34. Stahel PF, et al. Intrathecal levels of complement-derived soluble membrane attack complex (sC5b-9) correlate with blood-brain barrier dysfunction in patients with traumatic brain injury. J Neurotrauma. 2001;18(8):773-781.

35. Bellander BM, Singhrao SK, Ohlsson M, Mattsson P, Svensson M. Complement activation in the human brain after traumatic head injury. J Neurotrauma. 2001;18(12):1295-1311.

36. Rutkowski MJ, et al. Complement and the central nervous system: emerging roles in development, protection and regeneration. Immunol Cell Biol. 2010;88(8):781-786.

37. Derer S, Beurskens FJ, Rosner T, Peipp M, Valerius T. Complement in antibody-based tumor therapy. Crit Rev Immunol. 2014;34(3):199-214.

38. Rekate HL. The definition and classification of hydrocephalus: a personal recommendation to stimulate debate. Cerebrospinal Fluid Res. 2008;5:2.

39. Winston KR, Ho JT, Dolan SA. Recurrent cerebrospinal fluid shunt infection and the efficacy of reusing infected ventricular entry sites. J Neurosurg Pediatr. 2013;11(6):635-642.

40. Tamber MS, et al. Pediatric hydrocephalus: systematic literature review and evidence-based guidelines. Part 8: Management of cerebrospinal fluid shunt infection. J Neurosurg Pediatr. 2014;14 Suppl 1: 60-71.

41. Garton HJ, Kestle JR, Drake JM. Predicting shunt failure on the basis of clinical symptoms and signs in children. $J$ Neurosurg. 2001;94(2):202-210

42. Sivaganesan A, Krishnamurthy R, Sahni D, Viswanathan C. Neuroimaging of ventriculoperitoneal shunt complications in children. Pediatr Radiol. 2012;42(9):1029-1046.

43. Boyle TP, Nigrovic LE. Radiographic evaluation of pediatric cerebrospinal fluid shunt malfunction in the emergency setting Pediatr Emerg Care. 2015;31(6): 435-440; quiz 441-443.

44. Bayston R. Serum C-reactive protein test in diagnosis of septic complications of cerebrospinal fluid shunts for hydrocephalus. Arch Dis Child. 1979;54(7):545-548

45. Asi-Bautista MC, Heidemann SM, Meert KL, Canady AI, Sarnaik AP. Tumor necrosis factor-alpha, interleukin-1 beta, and interleukin-6 concentrations in cerebrospinal fluid predict ventriculoperitoneal shunt infection. Crit Care Med. 1997;25(10):1713-1716.

46. Heep A, et al. Vascular endothelial growth factor and transforming growth factor-betal are highly expressed in the cerebrospinal fluid of premature infants with posthemorrhagic hydrocephalus. Pediatr Res. 2004;56(5):768-774.

47. Schuhmann MU, et al. The value of C-reactive protein in the management of shunt infections. J Neurosurg. 2005; 103(3 Suppl):223-230

48. Lolak S, Bunyaratavej K. C-reactive protein in prediction of ventriculoperitoneal shunt-related infection in high-risk patients Surg Infect (Larchmt). 2013;14(2):192-195.

49. Morales DM, et al. Cerebrospinal fluid levels of amyloid precursor protein are associated with ventricular size in post-hemorrhagic hydrocephalus of prematurity. PloS One. 2015;10(3):e0115045.

50. Viraraghavan R, Jantausch B, Campos J. Late-onset central nervous system shunt infections with Propionibacterium acnes: diagnosis and management. Clin Pediatr (Phila). 2004;43(4):393-397.

51. Conen A, et al. Characteristics and treatment outcome of cerebrospinal fluid shunt-associated infections in adults: a retrospec tive analysis over an 11-year period. Clin Infect Dis. 2008;47(1):73-82.

52. Perry A, Lambert P. Propionibacterium acnes: infection beyond the skin. Expert Rev Anti Infect Ther. 2011;9(12):1149-1156

53. Stevens NT, et al. Ventriculoperitoneal shunt-related infections caused by Staphylococcus epidermidis: pathogenesis and implications for treatment. Br J Neurosurg. 2012;26(6):792-797. 
54. Achermann Y, Goldstein EJ, Coenye T, Shirtliff ME. Propionibacterium acnes: from commensal to opportunistic biofilm-associated implant pathogen. Clin Microbiol Rev. 2014;27(3):419-440.

55. Holland C, et al. Proteomic identification of secreted proteins of Propionibacterium acnes. BMC Microbiol. 2010;10:230.

56. Laarman A, Milder F, van Strijp J, Rooijakkers S. Complement inhibition by gram-positive pathogens: molecular mechanisms and therapeutic implications. J Mol Med (Berl). 2010;88(2):115-120.

57. Thammavongsa V, Kim HK, Missiakas D, Schneewind O. Staphylococcal manipulation of host immune responses. Nat Rev Microbiol. 2015;13(9):529-543.

58. Harboe M, Thorgersen EB, Mollnes TE. Advances in assay of complement function and activation. Adv Drug Deliv Rev. 2011;63(12):976-987.

59. Nilsson B, Ekdahl KN, Mollnes TE, Lambris JD. The role of complement in biomaterial-induced inflammation. Mol Immunol. 2007;44(1-3):82-94

60. Morgan BP, Gasque P. Expression of complement in the brain: role in health and disease. Immunol Today. 1996;17(10):461-466

61. Francis K, Van Beek J, Canova C, Neal JW, Gasque P. Innate immunity and brain inflammation: the key role of complement. Expert Rev Mol Med. 2003;5(15):1-19.

62. Stahel PF, Barnum SR. The role of the complement system in CNS inflammatory diseases. Expert Rev Clin Immunol. 2006;2(3):445-456. 